\title{
Clinical profiles of Chinese patients with diffuse panbronchiolitis
}

Kenneth W T Tsang, Clara G C Ooi, Mary S M Ip, Wah-kit Lam, Henry Ngan, Eric Y T Chan, Brian Hawkins, Chu-shak Ho, Ryoichi Amitani, Eisaku Tanaka, Harumi Itoh

Department of

Medicine

K W T Tsang

M S M Ip

W-k Lam

C-s Ho

Department of Diagnostic Radiology C G C Ooi

H Ngan

Department of Pathology

E Y T Chan

B Hawkins

Queen Mary Hospital, The University of Hong Kong, Pokfulam, Hong Kong

Chest Disease

Research Institute,

Kyoto University,

Japan

R Amitani

E Tanaka

$\mathrm{H}$ Itoh

Correspondence to: Dr K W T Tsang.

Received 24 July 1997 Returned to authors 18 December 1997 Revised version received 6 January 1998

Accepted for publication

7 January 1998
Abstract

Background-Diffuse panbronchiolitis (DPB), characterised by progressive sinobronchial sepsis, is well characterised in Japanese subjects but not in other ethnic groups. The experience with DPB in seven Chinese patients is described and the clinical profiles compared with those of Japanese subjects.

Methods-Seven Chinese patients (three women; mean (SD) age 48(18.6) years, all never smokers) who attended a teaching hospital centre and fulfilled the diagnostic criteria for DPB were assessed prospectively for clinical, radiological, lung function, microbiological, and other "characteristic" laboratory parameters.

Results-Lung function assessment showed a typical obstructive pattern $(n=5)$ and air trapping $(n=7)$. Typical bronchiolar infiltration by lymphocytes and plasma cells and accumulation of foamy macrophages in the intraluminal tissue were detected in open lung biopsy specimens $(n=2)$. Chest radiographs and high resolution computed tomographic scans revealed hyperinflation, diffuse nodules, bronchial thickening and dilatation, peripheral hypoattenuation, and bronchiolectasis. Radiological improvement, manifest as a reduction in nodular density and bronchial thickening, and persistence of other abnormalities such as air trapping were not accurately depicted by the classical Nakata or Akira classifications. The other "characteristic" features such as HLA-B54, IgG subclass deficiency, raised CD4/CD8 $T$ lymphocyte ratio, cold haemagglutinaemia, raised IgA, IgG, and rheumatoid factor were not present. Treatment with erythromycin led to excellent responses in symptoms, lung function indices, and the radiological picture. A review of the non-Japanese cases in the literature reveals that this absence of typical "additional features" in DPB might also be applicable to non-Japanese patients.

Conclusions-We report the only series of non-Japanese Mongoloid patients with well characterised DPB who had uncharacteristic investigation profiles. This experience should help other clinicians in the investigation and management of DPB in non-Japanese patients.

(Thorax 1998;53:274-280)
Keywords: diffuse panbronchiolitis; chronic bronchial sepsis; macrolides; Chinese

Diffuse panbronchiolitis (DPB) is a relatively newly recognised idiopathic chronic progressive suppurative airways disease originally described in Japan on necropsy cases. ${ }^{1}$ It typically presents with wheezing, chronic bronchial sepsis, and often rapidly progresses to respiratory failure and death before the introduction of low dose erythromycin therapy. ${ }^{2}$ Pathologically, thickening of the bronchiolar wall due to infiltration by lymphocytes, plasma cells and histiocytes, and peribronchiolar accumulation of foamy macrophages occur. ${ }^{3}$ DPB is distinct from asthma, bronchiectasis, and chronic obstructive pulmonary disease pathologically and radiologically although some of the clinical symptoms can overlap. ${ }^{4}$

Although DPB is not an uncommon disease amongst Japanese subjects, only sporadic cases have been reported in Caucasians, ${ }^{5-8}$ Koreans, ${ }^{910}$ Indians, ${ }^{11}$ black subjects, ${ }^{12}$ and Hispanics. ${ }^{5}{ }^{13}$ HLA-B54 is found in $63.2 \%$ of Japanese patients with DPB (relative risk 13.3) and has been reported in a widely quoted study to be prevalent in $10.4 \%$ of Chinese subjects. ${ }^{14}$ This suggests that the Chinese might also be susceptible to developing DPB. However, there has been no systematic study on DPB in Chinese subjects in whom it is still rarely reported and poorly defined. ${ }^{45-18}$ Underrecognition of this condition, which mimics primary ciliary dyskinesia, bronchiectasis, and cystic fibrosis, ${ }^{4}$ has serious consequences as DPB is highly responsive to treatment with low doses of erythromycin but is fatal if untreated. ${ }^{2}$ Although firmly established, the diagnostic criteria for DPB were constructed according to the characteristics of Japanese patients and have not been validated for other ethnic groups. ${ }^{419}$ We therefore report our experience with DPB in seven well characterised Chinese patients and compare the clinical profiles of our patients with those of the Japanese.

\section{Methods}

PATIENT POPULATION

Between October 1994 and October 1996 seven patients (pure southern Chinese) were diagnosed to have DPB at the Department of Medicine, University of Hong Kong. Diagnosis was made according to established diagnostic criteria $^{3-519}$ and after consultation with clinicians and radiologists experienced in DPB. An informal survey through the clinical practice of 
Table 1 Diagnostic criteria for diffuse panbronchiolitis

\begin{tabular}{l}
\hline Symptoms \\
Cough \\
Sputum production \\
Dyspnoea on exertion \\
Signs \\
Wheezing \\
Crackles \\
Radiology \\
Diffusely disseminated fine nodular shadow \\
Lower zone distribution \\
Hyperinflation \\
Lung function parameters \\
FEV $1<70 \%$ predicted or FEV $/$ FVC $<70 \%$ \\
VC $<80 \%$ predicted \\
RV $>150 \%$ predicted \\
PaO ${ }_{2}<80$ mm Hg $(\sim 10.6 \mathrm{kPa})$ \\
Additional "characteristic but non-diagnostic" features \\
Chronic paranasal sinusitis \\
Increased serum cold haemagglutinin without raised \\
anti- Mycoplasma pneumoniae antibody \\
Increased serum IgA and IgG \\
Increased CD4/CD8 lymphocyte ratio \\
Proof of HLA Bw54 antigen \\
Positive rheumatoid factor
\end{tabular}

$\mathrm{FEV}_{1}=$ forced expiratory volume in one second; $\mathrm{FVC}=$ forced vital capacity; $\mathrm{VC}=$ vital capacity; $\mathrm{RV}=$ residual volume; $\mathrm{PaO}_{2}=$ arterial oxygen tension.

specialist members of the Hong Kong Thoracic Society did not reveal any other cases in Hong Kong.

DIAGNOSTIC FEATURES

All patients presented with typical symptoms, signs, and plain radiographic and thoracic high resolution computed tomographic (HRCT) features that fulfilled the clinical diagnostic criteria adopted by the Japanese National Survey ${ }^{19}$ and others as outlined in table $1 .^{3-5}$ All patients presented with chronic cough, sputum production, and dyspnoea on exertion, while four patients had end inspiratory crackles and five had wheezes in their chest at initial presentation. All but one patient had nasal symptoms (obstruction and recurrent chronic discharge) confirmed by expert otolaryngologists to be chronic sinusitis. The other patient details are shown in tables 2 and 3. Histological data were available for three patients (patients 6 and 7 both had open lung and transbronchial biopsies and patient 1 had a transbronchial biopsy only) which were based on the pathological diagnostic criteria outlined by Iwata et al. ${ }^{4}$ The most important features were the presence of respiratory bronchiolitis and peribronchiolitis with bronchiolar narrowing from infiltration of mononuclear cell infiltration and/or granulation tissue, and the presence of foam cells in the bronchioles. ${ }^{4}$ The transbronchial biopsy specimens were compatible with the diagnostic criteria in patients 6 and 7 (also confirmed by open lung biopsy specimens in both cases), but were non-diagnostic in patient 1 (who declined open lung biopsy). Patients 2 and 4 refused whilst patients 3 and 5 were considered too unstable to undergo any invasive procedures.

\section{INVESTIGATION PROFILES}

Lung function indices were measured using a Sensor-Medics 2200 Lung Function package and standard protocol as per routine clinical practice. Nasal mucosa was obtained from the inferior turbinate of subjects with a cytology brush (without anaesthetic) and resuspended in medium 199 (Flow Laboratory, Paisley, Scotland, UK) before examination of ciliary movement and beat frequency with a Leica DM LB phase contrast microscope (Leica, Wetzlar, Germany) and a MPV-COMBI (Leica, Wetzlar, Germany) photomultiplier system as described previously. ${ }^{20}$ Ciliated epithelium was fixed in $2.5 \%$ glutaraldehyde (in osmium tetroxide buffer) and embedded in araldite for ultrastructural examination by a trained electron microscopy technician. ${ }^{21}$ Evaluation of routine haematological indices and renal and liver biochemical profiles; serum immunoglobulin (Ig)G, IgA, and IgM; autoantibody titres (for rheumatoid factor, antinuclear factor, and IgG against $\mathrm{Ro}, \mathrm{La}, \mathrm{Jo}_{1}$, mitochondrial, and smooth muscle); arterial blood gas tensions; serum IgG subclasses; lymphocyte subset analysis; $\alpha_{1}$-antitrypsin level; viral titres (measles, mumps, influenza, parainfluenza, respiratory syncytial, adenovirus, rotavirus, and enterovirus); blood CD4/CD8 lymphocyte ratio; IgG against Pseudomonas

Table 2 Characteristics of patients at presentation

\begin{tabular}{|c|c|c|c|c|c|c|c|}
\hline & \multicolumn{7}{|l|}{ Patient no. } \\
\hline & $1(M)$ & $2(M)$ & $3(F)$ & $4(M)$ & $5(M)$ & $6(F)$ & $7(F)$ \\
\hline Age at onset (years) & 54 & 28 & 32 & 60 & 63 & 72 & 27 \\
\hline Age at assessment (years) & 57 & 30 & 32 & 61 & 64 & 73 & 33 \\
\hline No. of months on treatment & 28 & 14 & 4 & 4 & 4 & 3 & 2 \\
\hline Nasal symptoms (years) & 30 & 15 & 15 & None & 50 & 15 & 10 \\
\hline Physical examination & Wheezes & Normal & Wheezes/crackles & Wheezes/crackles & Wheezes/crackles & Wheezes/crackles & Wheezes \\
\hline Sputum pathogen & H influenzae & $P$ aeruginosa & Commensals & H influenzae & $P$ aeruginosa & $\begin{array}{l}\text { Mycobacterium } \\
\text { tuberculosis }\end{array}$ & H influenzae \\
\hline Travel history to Japan & Nil & 15 years before & Nil & Nil & 11 years before & Nil & $\begin{array}{l}10 \text { years } \\
\text { before }\end{array}$ \\
\hline Medication & Atenolol & Nil & Nil & Nil & Nil & Nil & Nil \\
\hline Concurrent illness & Hypertension & Renal transplant & Nil & Nil & Cor pulmonale & Neurofibroma T8 & Nil \\
\hline \multicolumn{8}{|l|}{ Ciliary beat frequency } \\
\hline$(12-16 \mathrm{~Hz})^{\star}$ & 12 & 12.1 & 9.7 & 14.1 & 14.1 & 12.2 & 10.1 \\
\hline Ciliary ultrastructure & Normal & Normal & Normal & Normal & Normal & Normal & Normal \\
\hline Serum IgA $(90-450 \mathrm{mg} / \mathrm{dl})^{\star}$ & 268 & 224 & 614 & 420 & 368 & 365 & 465 \\
\hline $\mathrm{CD} 4 / \mathrm{CD} 8$ ratio $(0.63-3.24)^{\star}$ & 1.98 & 0.57 & 3.55 & 3.7 & 1.58 & 0.78 & 1.88 \\
\hline Cold haemagglutinin & Negative & Negative & Negative & Negative & Negative & Negative & Negative \\
\hline IgG against $M$ pneumoniae & Negative & Negative & Negative & Negative & Negative & Negative & Negative \\
\hline \multirow[t]{2}{*}{ HLA typing } & $\mathrm{A} 2, \mathrm{~B} 5, \mathrm{~B} 60$ & $\mathrm{~A} 2, \mathrm{~A} 33, \mathrm{~B} 17$ & $\mathrm{~A} 24, \mathrm{~A} 11, \mathrm{~B} 13$, & $\mathrm{A} 2, \mathrm{~A} 24, \mathrm{~B} 55$ & $\mathrm{~A} 24, \mathrm{~B} 60, \mathrm{~B} 35$ & $\mathrm{~A} 2, \mathrm{~A} 11, \mathrm{~B} 60$ & $\mathrm{~A} 24, \mathrm{~A} 11, \mathrm{~B} 27$ \\
\hline & DR9,DR11 & B55,DR15,DR3 & B51,DR9,DR16 & B70,DR9,DR16 & DR9,DR12 & DR8,DR9 & DR12,DR15 \\
\hline Histological examination & TBB & Nil & Nil & Nil & Nil & $\mathrm{TBB}+\mathrm{OLB}$ & TBB+OLB \\
\hline
\end{tabular}

$\mathrm{TBB}$ and $\mathrm{OLB}=$ transbronchial and open lung biopsies, respectively.

*Normal range for data. 
Table 3 Investigation profiles at presentation and re-assessment

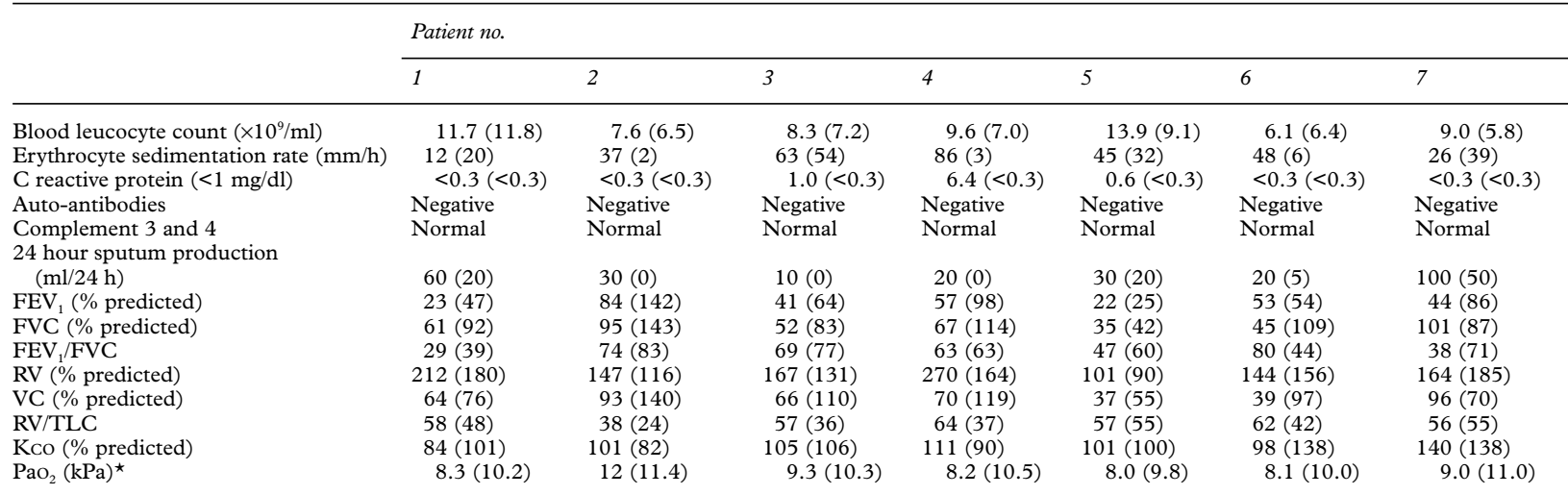

Data shown are measurements made at presentation and re-assessment (in parentheses).

*Normal range for arterial blood gases when breathing room air $10.6-14 \mathrm{kPa}$.

$\mathrm{FEV}_{1}=$ forced expiratory volume in one second; $\mathrm{FVC}=$ forced vital capacity; $\mathrm{RV}=$ residual volume; $\mathrm{VC}=$ vital capacity; TLC=total lung capacity; $\mathrm{KcO}=$ carbon monoxide transfer coefficient; $\mathrm{PaO}_{2}=$ arterial oxygen tension.

pseudomallei, Legionella pneumophilia, Chlamydia psittaci, Mycoplasma pneumoniae, and human adult $\mathrm{T}$ cell leukaemia virus (HTLV-1); Aspergillus precipitins; serum cold haemagglutinin; complements 3 and 4; sputum microbiology (routine aerobic and anaerobic, mycobacterial and fungal cultures), and full human leucocyte antigen (HLA) typing were performed at the Biochemistry, Haematology, Clinical Immunology, Microbiology, and Tissue Typing departments of the University of Hong Kong using routine methods. Density gradient sedimentation with Lymphoprep $(\mathrm{Ny}-$ comed, Oslo, Norway) and ammonium chloride were used to remove the mononuclear and red cells in leucocyte-rich concentrate obtained from heparinised blood mixed with $6 \%$ dextran. Cytospins of granulocytes were fixed in absolute alcohol at $4^{\circ} \mathrm{C}$ for five minutes. Serum samples were diluted $(1: 20)$ in phosphate buffered saline and anti-neutrophil cytoplasmic antibody (ANCA) binding was detected by rabbit anti-human IgG-FITC conjugate (Dako, Glostrup, Denmark). ${ }^{22}$

RADIOLOGICAL ASSESSMENT

All seven patients underwent thoracic HRCT scanning at the time of diagnosis and reassessment (after 8.6 (9.4) months of erythromycin therapy) and were scanned in the supine position (scan thickness $1 \mathrm{~mm}$ or $1.5 \mathrm{~mm}$ and slice interval $10 \mathrm{~mm}$ ). Expiratory scans used to evaluate air trapping were obtained for two patients before and seven patients after erythromycin therapy. The HRCT scan and plain postero-anterior films were analysed by three experienced radiologists who resolved differences by consensus. Each lung was divided into three zones: upper (apex to carina), middle (carina to venous confluence), and lower (venous confluence to bases). Six lung zones were therefore evaluated individually for each patient. HRCT scans were graded as described previously by Akira et $a^{23}$ : type 1, nodules associated with bronchovascular branchings; type 2 , nodules branching in appearance ("tree-inbud"); type 3, nodules connected to ringshaped or ductal opacities (bronchiolectasis); and type 4, dilatation of proximal terminal bronchioles and bronchi (predominantly at the peripheral airways). ${ }^{23}{ }^{24}$ Distribution (diffuse or localised), symmetry, profusion of nodules, and areas of hypoattenuation and air trapping (on expiratory HRCT scans) were also assessed. Grading for nodular profusion, hypoattenuation, and air trapping was made according to the extent of involvement in each lung zone as: 0 (normal), 1 ( $<25 \%$ of lung zone involvement), 2 ( $>25 \%$ but $<50 \%$ ), and $3(>50 \%)$. Bronchial dilatation was similarly graded by comparing bronchial calibre with that of the accompanying artery: 0 (normal bronchial calibre), 1 (<1.5 times calibre of accompanying artery), 2 ( $>1.5$ but $<2.0$ times), and $3(>2$ times). Bronchial thickening was graded as 1 (mild), 2 (moderate), and 3 (severe). For each

Table 4 Radiological assessment at presentation and re-assessment

\begin{tabular}{|c|c|c|c|c|c|c|c|}
\hline & \multicolumn{7}{|l|}{ Patient no. } \\
\hline & 1 & 2 & 3 & 4 & 5 & 6 & 7 \\
\hline Nakata radiographic grading ${ }^{23}$ & III (III) & II (II) & III (III) & IV (II) & IV (IV) & III (III) & II (II) \\
\hline Akira HRCT grading ${ }^{22} 29$ & $3(4)$ & $3(2)$ & $3(3)$ & $3(2)$ & $4(4)$ & $2(2)$ & $3(3)$ \\
\hline Nodular profusion, overall score & $2(0.7)$ & $1.8(0.8)$ & $2(1.7)$ & $2.3(1.3)$ & $2.3(2)$ & $2.7(1.4)$ & $1.7(1.2)$ \\
\hline Nodular symmetry & Asymmetrical & Asymmetrical & Symmetrical & Symmetrical & Symmetrical & Symmetrical & Symmetrical \\
\hline Nodular distribution & Diffuse & Diffuse & Diffuse & Diffuse & Diffuse & Diffuse & Diffuse \\
\hline Airway dilatation, overall score & $1.5(1.8)$ & $1.0(0.8)$ & $1.1(0.8)$ & $0.8(0.3)$ & $2.0(2.0)$ & $1.6(1.0)$ & $0.7(0.7)$ \\
\hline Airway thickening & $1.5(1.3)$ & $1.0(0.8)$ & $1.3(0.7)$ & $0.7(0.3)$ & $1.3(1.3)$ & $2.0(0.4)$ & $1.7(1.3)$ \\
\hline Hypoattenuation, overall score & $2(3)$ & $0.2(0.2)$ & $0.8(1.2)$ & $0.2(0.2)$ & $1.7(2.7)$ & $1.7(2.6)$ & $1.0(1.0)$ \\
\hline Air trapping,$^{\star}$ overall score & $\mathrm{NP}(1.2)$ & $1.3(1)$ & $\mathrm{NP}(1.3)$ & $\mathrm{NP}(1.0)$ & $2.0(2.7)$ & NP (2.6) & $\mathrm{NP}(0.7)$ \\
\hline
\end{tabular}

Data shown are assessments made at presentation and re-assessment (in parentheses).

*Assessment made at expiratory HRCT scanning of the thorax.

$\mathrm{NP}=$ not performed

Please refer to text for the scoring systems. 


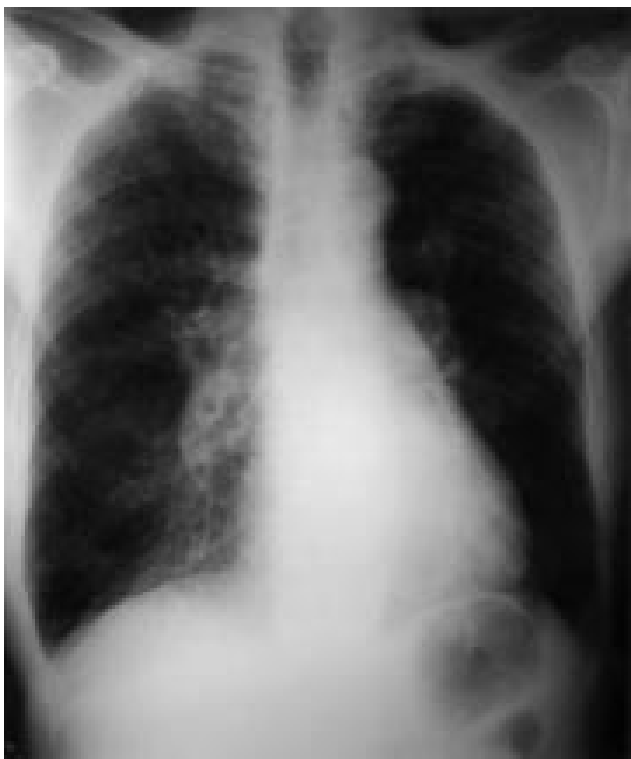

Figure 1 Plain chest radiograph in case 1 showing multiple diffuse nodules in both lung fields with a predominance at the right hemithorax. There was also hyperinflation consistent with air trapping.

of these features an overall score was obtained by dividing the sum of the scores from all lung zones by six.

Chest radiographs corresponding in time to the HRCT scans were graded from types I to V as described by Nakata et al. ${ }^{24}$ Types I, II, III, IV, and $\mathrm{V}$ were assigned when there was overinflation without nodular shadows, disseminated small nodular shadows confined to one lung, small nodular shadows in both lungs, ring-shaped or tramline shadows in the lower lungs with nodular shadows, and large ringshaped and nodular shadows, respectively.

\section{Results}

DEMOGRAPHIC DATA

The mean (SD) ages at onset of symptoms and at initial assessment were 48 (18.6) and 50 (17.8) years. None of the seven patients (three women) had ever smoked cigarettes and they received treatment with erythromycin $(250 \mathrm{mg}$ twice daily) for 8.6 (9.4) months before re-assessment. Three patients had travelled to Japan 15, 11, and 10 years before the onset of symptoms (table 2 ).
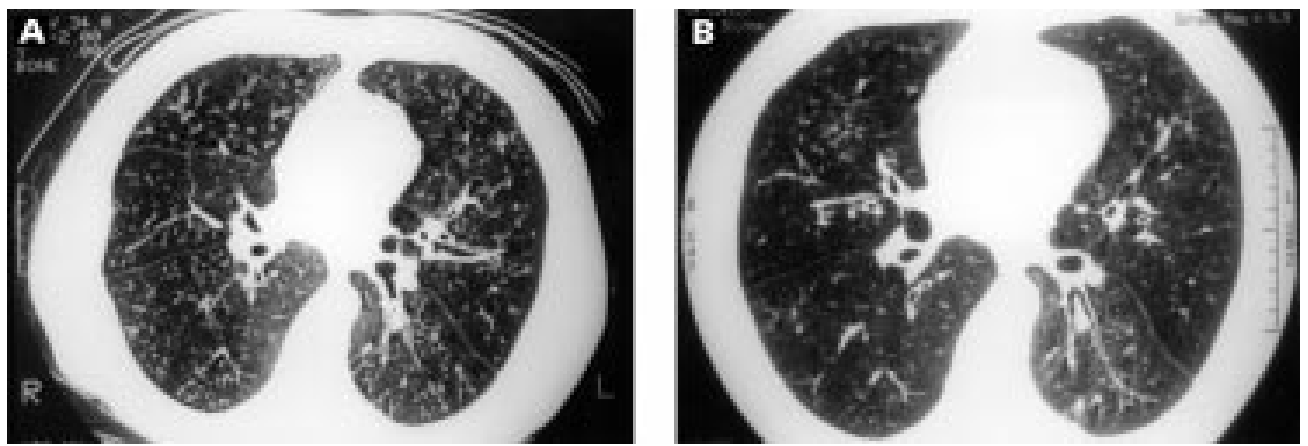

CLINICAL FEATURES

Six patients had had nasal symptoms (obstruction and rhinorrhoea) for a mean of 22.5 (15.1) years. All seven patients presented with copious purulent sputum production $(38.6(31.2) \mathrm{ml} /$ 24 hours), cough, and dyspnoea on exertion. At the initial assessment six patients were found to have wheezes and five had crackles in their chest on physical examination. After 8.6 (9.4) months of treatment with erythromycin the presenting symptoms of dyspnoea on exertion, cough, sputum production, and nasal symptoms improved in all seven patients. The mean sputum volume reduced to 13.6 (18.4) $\mathrm{ml} / 24$ hours and patients 2,3 , and 4 had no further sputum production at re-assessment (table 3). Examination of the chest revealed no further wheezing in five and persistence of crackles in five patients.

INVESTIGATION PROFILES

All the patients underwent the investigation profiles listed above and repeated measurements were made on some selected parameters as shown in table 3. At initial presentation the mean (SD) forced expiratory volume in one second $\left(\mathrm{FEV}_{1}\right)$, forced vital capacity (FVC), residual volume (RV), vital capacity (VC), carbon monoxide transfer coefficient (KCO), and arterial oxygen tension $\left(\mathrm{PaO}_{2}\right)$ were 46.3 (21.4)\%, 65.1 (24.8)\%, $172.1(54.4) \%, 66.4$ (23.2)\%, $105.7(17.2) \%$, and $9.0(1.4) \mathrm{kPa}$, respectively, which changed to $73.7(38.7) \%$, 95.7 (31.3)\%, $146(35.0) \%, 95.3(30.0) \%$, $107.9(22.1) \%$, and $10.5(0.6) \mathrm{kPa}$ after erythromycin therapy. Tests for ciliary beat movement and frequency, ciliary ultrastructure, HLA typing, $\alpha_{1}$-antitrypsin, viral titres, IgG against $P$ pseudomallei, $L$ pneumophilia, $C$ psittaci, $M$ pneumoniae, and HTLV-1, Aspergillus precipitins, complements 3 and 4, and routine haematological and biochemical indices were negative or normal at initial presentation. Serum $\operatorname{IgA}$ and $\mathrm{IgG}_{3}$ levels were persistently raised in two and three patients, respectively, although the levels of other immunoglobulins were normal. Antinuclear factor was positive in six patients and anti-smooth muscle in two, while anti-dsDNA, anti-extractable nuclear antigen, rheumatoid factor, and anti-mitochondrial antibodies were negative in all patients. ANCA (cytoplasmic) was positive in six patients, none of whom had anti-proteinase

Figure 2 Thoracic high resolution computed tomographic scans in case 1 showing $(A)$ centrilobular nodules with tree-in-bud appearance in the lower lobes and bronchial thickening and dilatation at the onset of diffuse panbronchiolitis, and $(B)$ significant resolution of the nodular densities after starting treatment with erythromycin. 
III antibody. Lymphocyte subset distribution was normal in three patients but the remaining four displayed an increase in CD4 $\mathrm{T}$ cells, a decrease in CD3 $\mathrm{T}$ cells, an increase in CD3 and CD8 T cells, and a decrease in CD 3 and CD8 $\mathrm{T}$ cells, respectively. The CD4/CD8 ratio was raised in two patients, reduced in one, and normal in four patients.

$P$ aeruginosa was isolated from the sputum of two patients and Haemophilus influenzae from three patients at initial presentation. The remaining case had no identifiable pathogen in her sputum. The sputum of patient 6 yielded only Mycobacterium tuberculosis on culture (negative Ziehl-Neelson staining), although repeated bronchoscopic, bronchial and transbronchial biopsies and bronchoalveolar lavage had not yielded any other evidence of tuberculous infection (endobronchial and parenchymal). In addition, a two month supervised course of rifampicin, isoniazid, ethambutol, and pyrazinamide did not alter her symptoms or produce any improvement in lung function or radiology (HRCT and plain radiography). This was followed by open lung biopsy which confirmed the diagnosis of DPB.

RADIOLOGICAL ASSESSMENT

At the initial assessment nodular distribution with lower lobe predominance was bilateral and diffuse in all except two cases, in whom it was asymmetrical (table 4). After treatment with erythromycin there was an overall improvement in both bronchial thickening and dilatation, but not in hypoattenuation of the peripheral areas. Air trapping was confirmed on expiratory scans in only two cases at initial presentation and in all seven cases after erythromycin treatment. The serial Akira and Nakata classifications remained unchanged in four and six patients, respectively, and did not appear to reflect the reduction in nodular perfusion which was the most significant response to treatment (figs 1 and 2).

\section{Discussion}

All our patients presented with typical chronic sinobronchial sepsis and most had persistence of $P$ aeruginosa or $H$ influenzae in their sputum, characteristic radiological features, and excellent clinical and radiological response to macrolide therapy (tables 1-4). However, our cases differ from the Japanese patients (table 1) in several respects, primarily the absence of HLA-B54 type. The Chinese patient mentioned briefly by Iwata et al did not possess HLA-B54 but shared a common feature with most of our patients in possessing HLA-A2. ${ }^{4}$ The $10 \%$ prevalence of HLA-54 in Chinese subjects quoted in the literature ${ }^{14}$ does not agree with our finding of a prevalence of $3 \%$ in patients from southern China. ${ }^{25}$ If HLA-B54 has a pathological role in DPB, then this low incidence of HLA-54 would explain the relative scarcity of DPB in the Chinese population, in addition to the probable underdiagnosis and reporting. Five of the seven patients in this series had HLA-DR9 compared with $29.2 \%$ in the normal population in Hong Kong. Although the number of patients tested is small, our preliminary results suggest that future patients should also be tested for HLADR9 and HLA-A2. Two of our patients also had HLA-B55, which has also been reported to be increased in a Japanese study. ${ }^{14}$

Three of our cases had raised $\mathrm{IgG}_{3}$ levels but $\mathrm{IgG}_{4}$ levels were normal, whereas $30.8 \%$ of Japanese patients were reported to have $\mathrm{IgG}_{4}$ deficiency. ${ }^{26}$ The other "atypical" features include normal or low CD4/CD8 lymphocyte ratio in five patients, absence of raised serum $\operatorname{IgA}$ in five, and absence of raised serum $\operatorname{IgG}$, cold haemagglutinin and rheumatoid factor in seven patients. ${ }^{5}$ A review of the cases in the literature of DPB in non-Japanese subjects revealed a total of 19 Mongoloid (14 Chinese and five Koreans) and 13 non-Mongoloid cases who generally had not been systematically evaluated. $^{4-81012} 1316-1827$ Of these, HLA-B54 was detected in only two cases in the former and either not checked or not detected in the latter group. In addition, raised levels of cold haemagglutinin were found in two Hispanic patients but not in the rest of the aforementioned 32 cases. $^{513}$ The other investigation profiles that were considered to be characteristic and helpful in the diagnosis (table 1), including CD4/CD8 lymphocyte ratio, serum IgA level, IgG subclass analysis, and $M$ pneumoniae serology, were not evaluated in these non-Japanese patients. Our results suggest that these "additional" features (table 1) might not be applicable in the diagnosis of DPB in non-Japanese patients. The finding of a positive ANCA in six of our patients is also interesting and needs confirmation by other workers, as this has not been reported previously in DPB.

The value of HRCT scans in assessing small airways disease in DPB is clear and is substantiated by pathological-radiological correlation. ${ }^{23} 2829$ This experience, however, is limited to Japan, largely due to the relative confinement of DPB to that country. Peripheral areas of hypoattenuation on HRCT scanning $^{29-31}$ are a non-specific indicator of air trapping and reflect an underlying hyperinflation caused by narrowing of the small airways. ${ }^{29}$ In the only other series of patients with DPB followed up by HRCT scanning after treatment with erythromycin there was an improvement in nodular profusion but not airway dilatation and peripheral hypoattenuation. ${ }^{30}$ In contrast, our findings suggest that there was partial reversibility in bronchiolar dilatation in our patients, although no corresponding improvement in the peripheral areas of hypoattenuation was evident. This might be due to selective and more severe injury to the peripheral airways resulting in sustained distal air trapping, whilst the less affected central larger airways recovered with erythromycin treatment. However, in agreement with Akira et al, ${ }^{30}$ there was a universal reduction in nodular profusion in all our patients after erythromycin treatment. This important feature in the radiological follow up of these patients, which appears to parallel the improvement in lung function and clinical improvement, could not be accurately represented in the Nakata 
classification. ${ }^{28}$ Our study also highlights the relative insensitivity of plain radiography compared with HRCT scanning in assessing disease activity, particularly in small airway pathology. The usefulness of the Nakata radiographic and Akira HRCT classifications in this study therefore appears to be limited.

As with Japanese patients, our patients responded very well in terms of lung function indices, blood oxygenation, symptomatic relief, sputum production, and radiological evaluation to low dose, long term treatment with erythromycin. $^{2}$ Whilst the efficacy of macrolides in treating DPB is well established, the mechanism(s) is still largely unknown. One possible mechanism is the reduction of pulmonary levels of interleukin 8 and leukotriene $\mathrm{B}_{4}$ which are potent chemotactic agents. ${ }^{32} 33$ This leads to a reduction in neutrophil influx into the airways and alveolar space ${ }^{34}$ which is known to cause airway destruction in chronic bronchial sepsis by various processes such as the release of human neutrophil elastase. ${ }^{35}{ }^{36}$ It is also possible that erythromycin interferes with the formation of $P$ aeruginosa biofilms which are important in its persistence in the airways. ${ }^{37}$ Until the precise mechanism is known, more specifically targeted treatment cannot be planned other than empirical treatment with macrolides. There appears to be a "class effect" in that most of the available macrolides including erythromycin, clarithromycin, roxithromycin, and azithromycin, are efficacious in DPB. ${ }^{39}$ For resistant cases recent in vitro and clinical evidence suggests that inhalation of indomethacin and oxitropium bromide might be effective in reducing sputum production. ${ }^{40} 41$

Interestingly, three of our patients had travelled to Japan prior to the onset of symptoms, similar to the Hispanic man who developed DPB after extensive travels to Japan and South East Asia. ${ }^{13}$ However, the long interval between the journey to Japan and the onset of symptoms makes an aetiological linkage very unlikely in our patients. As in the cases in the Japanese literature, there was no identifiable aetiology for the onset of chronic bronchial sepsis or DPB in our patients. Our investigations have also effectively excluded all the known differential diagnoses for DPB including primary ciliary dyskinesia, bronchiectasis, chronic bronchitis and emphysema, bronchiolitis obliterans, cystic fibrosis (not known to occur in Chinese), Wegener's granulomatosis, malignant lymphoma, and bare lymphocyte syndrome. ${ }^{4}$

Whilst we have demonstrated characteristic features of DPB in all our cases, only four patients were examined histologically for features of DPB. Open lung biopsy specimens of patients who fulfilled the clinical diagnostic criteria for DPB might very occasionally show unclassified bronchiolitis and bronchiolectasis. ${ }^{42}$ However, in most instances the pathological changes in DPB correlate well with HRCT features $^{29}$ and HRCT scanning is advocated and frequently used as the diagnostic tool in this condition, ${ }^{43}$ especially in Japan where clinicians and radiologists have considerable relevant experience. In practice, most patients with DPB in Japan are routinely diagnosed using high quality thoracic HRCT scans. The clinical and radiological response to erythromycin also gives very good circumstantial evidence to support the diagnosis of DPB.

We have reported seven cases of DPB who, despite typical clinical features, appear to have different investigation profiles from their Japanese counterparts. More experience needs to be gathered on non-Japanese patients to evaluate further the clinical characteristics of this macrolide responsive but otherwise progressive idiopathic pulmonary disease.

The authors wish to thank Drs Y P Tai, S W Pang, Maria P Wong, B Lam, W M Chan, S P Poon, J March, and M Lo for their invaluable help in the preparation of this manuscript.

1 Yamanaka A, Saiki S, Tamura S, et al. The problems of chronic obstructive pulmonary disease: especially concernCika 1996;23:442-51.

2 Kudoh S, Uteka T, Hagiwara K, et al. Clinical effects of low dose long term erythromycin chemotherapy of diffuse panbronchiolitis. Fpn f Thorac Dis 1987;25:632-42.

3 Sugiyama Y. Diffuse panbronchiolitis. In: King TE, ed. Clinics in Chest Medicine, Vol 14. Philadelphia: WB Saunders, 1993: 765-72.

4 Iwata M, Sata A, Colby T. Diffuse panbronchiolitis. In: Epler GR, ed. Diseases of the bronchioles. New York: Raven Press, 1994: 153-79.

5 Fitzgerald JE, King TE, Lynch DA, et al. Diffuse panbronchiolitis in the United States. Am f Respir Crit Care Med 1996;154:497-503.

6 Naalsund A, Foerster A, Aasebo U, et al. Svar pa efterlysning av diffus pabronchkiolit. Nu har den hittat hi! Lakartidningen 1995;92:3119-21.

7 Tredaniel J, Zalcman G, Gerber F, et al. Diffuse panbronchiolitis: efficacy of low-dose erythromycin. Respir Med 1993;87:229-30.

8 Poletti V, Patelli M, Poletti G, et al. Diffuse panbronchiolitis observed in an Italian man. Chest 1990;98:515-6.

9 Sugiyama Y, Takeuchi K, Yotsumoto $\mathrm{H}$, et al. A case of diffuse panbronchiolitis in a second generation Korean male. Nippon Kyobu Shikkan Gakkai Zasshi 1986;24:83-7.

$10 \mathrm{Kim}$ YW, Han SK, Shim YS, et al. The first report of diffuse panbronchiolitis in Korea: five case reports. Intern Med 1992;31:695-701.

11 Brugiere O, Milleron B, Antoine M, et al. Diffuse panbronchiolitis in an Asian immigrant. Thorax 1996;51:1065-7.

12 Bas MA, Kussin PS, van Trigt P, et al. Recurrence of diffuse panbronchiolitis after lung transplantation. Am $\mathcal{F}$ Respir Crit Care Med 1995;151:895-8

13 Homer RJ, Khoo L, Smith GJ. Diffuse panbronchiolitis in a Hispanic man with travel history to Japan. Chest 1995;107: 1176-8.

14 Sugiyama Y, Kudoh S, Maeda H, et al. Analysis of HLA antigens in patients with diffuse panbronchiolitis. Am Rev Respir Dis 1990;141:1459.

15 Tsang KWT, Lam WK, Ip MSM, et al. Diffuse panbronchiolitis in the United states. Am F Respir Crit Care Med 1997; 155:2114

16 Wang HD, Sun TY, Miao JZ. Diffuse panbronchiolitis diagnosed by open lung biopsy. Abstracts of the 4th Congress of the Asian Pacific Society of Respirology Beijing: 1996, 47.

7 Chern MS, Yuen KH, Hsu YT, et al. Radiology of diffuse panbronchiolitis: experience in VGH-Taipei. Chung Hua Hsueh Tsa Chih Taipei 1992;50:469-74.

18 Chu YC, Yeh SZ, Chen CL, et al. Diffuse panbronchiolitis: report of a case. F Formos Med Assoc 1992;91:912-5.

19 Homma H, Yamanaka A, Tanimoto S, et al. Diffuse panbronchiolitis: a disease of the transitional zone of the lung. Chest 1983;83:63-9.

20 Greenstone M, Logan-Sinclair R, Cole PJ. An automated method to measure ciliary beat frequency. IRCS Med Sci $1984 ; 12: 715-6$

21 Tsang KWT, Rutman R, Kanthakumar K, et al. Haemophilus influenzae infection of human respiratory mucosa in low concentrations of antibiotics. Am Rev Respir Dis 1993;148: $201-7$

22 Wiih A. Delineation of a standard procedure for indirect immunofluorescence detection of ANCA. Acta Pathol Microbiol Immunol Scand 1989;97(Suppl 6):12-3.

23 Akira M, Kitatani F, Lee YS, et al. Diffuse panbronchiolitis: evaluation with high resolution CT. Radiology 1988;168 433-8.

24 Nakata K, Tanimoto M. Diffuse panbronchiolitis. fpn 7 Clin Radiol 1981;26:1133-42.

25 Hawkins BR, Serjeantson SW, Higgins DA. Distribution and co-occurrence of MHC class I, II and III markers in Southern Chinese: implications for autoimmune disease. Disease Markers 1988;6:237-45.

26 Sugiyama Y, Kudoh S, Kurashima A, et al. Serum IgG subclasses in diffuse panbronchiolitis. Igaku no ayumi 1987; $142: 833$. 
27 Randawa P, Hoagland MH, Yousem SA. Diffuse panbron28 Nakata H, Kimoto T, Nakayama T, et al. Diffuse peripheral Nakata $\mathrm{H}$, Kimoto $\mathrm{T}$, Nakayama $\mathrm{T}$, et al. Diffuse peripheral
lung disease: evaluation by high resolution computed tomography. Radiology 1985;157:181-5.

29 Nishimura $K$, Kitaichi M, Izumi $T$, et al. Diffuse panbronchiolitis: correlation with high resolution computerized tomography and pathological findings. Radiology 1992;184:779-85.

30 Akira M, Higashihara $T$, Sakatani $M$, et al. Diffuse panbronchiolitis: follow up CT examination. Radiology 1993;189:559-62.

31 Murata $\mathrm{K}$, Itoh $\mathrm{H}$, Senda $\mathrm{M}$, et al. Stratified impairment of pulmonary ventilation in diffuse panbronchiolitis: PET and CT studies. 7 Comput Assit Tomogr 1989;13:48-53.

32 Koga T. Neutrophilia and high level of interleukin 8 in the bronchoalveolar lavage fluid of diffuse panbronchiolitis. bronchoalveolar lavage fluid
Kurume Med $\mathcal{F}$ 1993;40:139-46.

33 Oda H, Kadota J, Kohno S, et al. Leukotriene B4 in bronchoalveolar lavage fluid of patients with diffuse bronchoalveolar lavage fluid of patients

34 Oda H, Kadota J, Kohno S, et al. Erythromycin inhibits chemotaxis in bronchoalveoli of diffuse panbronchiolitis. Chest 1994;106:1116-23.

35 Cole PJ. Inflammation: a two edge-sword: the model of bronchiectasis. Eur F Respir Dis Suppl 1986;147:6-15.
36 Amitani R, Wilson R, Rutman A, et al. Effects of human neutrophil elastase and bacterial proteinases on human respiratory epithelium. Am 7 Respir Cell Mol Biol 1991;4: 26-32.

37 Tsang KWT, Rutman A, Tanaka E, et al. Interaction of Pseudomonas aeruginosa with human respiratory mucosa in vitro. Eur Respir f 1994;7:1746-53.

38 Takeda H, Oogaki N, Kikuchi N, et al. A study to clarify the usefulness of the macrolides - the influence of clarithromycin to biofilm with Pseudomonas aeruginosa. KansenshogakuZasshi 1992;66:1454-61.

39 Kobayashi H, Takeda H, Sakayori S, et al. Study on azithroKobayashi $\mathrm{H}$, Takeda $\mathrm{H}$, Sakayori $\mathrm{S}$, et al. Study on azithro-
mycin in treatment of diffuse panbronchiolitis. Kansenshogaku-Zasshi 1995;69:711-22.

40 Tamaoki J, Chiyotani A, Kobayashi K, et al. Effects of indomethacin on bronchorrhea in patient with chronic bronchitis, diffuse panbronchiolitis or bronchiectasis. Am Rev Respir Dis 1992;145:548-52.

41 Tamaoki J, Chiyotani A, Tagaya E, et al. Effects of long term treatment with oxitropium bromide on airway secretions in chronic bronchitis and diffuse panbronchiolitis. Thorax 1994;49:545-8.

42 Izumi T. Diffuse panbronchiolitis. Chest 1991;100:596-7.

43 Corne J. Diffuse panbronchiolitis: a new Japanese export? Lancet 1996;248:1456-7. 